\title{
Designing and manufacturing of the coil support structure of W7-X
}

\author{
Didier Chauvin(1), Torsten Koppe(1), Antonio Cardella(1), Bernd Missal(1), Dirk Pilopp(1),
}

Jens Reich(1), Giovanni Di Bartolo(2), 'Rocio Camín, Ivan Gonzales (3), Luca Giordano(4) and Stefano Langone (5).

1. Max-Planck-Institut für Plasmaphysik, EURATOM Association, Teilinstitut Greifswald, Wendelsteinstr. 1, D-17491 Greifswald, Germany

2. M\&G srl Consultants, Via Dei Romanelli 8, I-20034 Giussano, Italy

3. Equipos Nucleares S.A., Avda. Juan Carlos 1, 39600 Maliano, Spain

4. Rovera Construzioni Meccaniche, Via Vecchia di Cuneo, Italy

5. Romabau Gerinox AG, Fohlenweide, $\mathrm{CH}-8570$ Weinfelden/Thurgau, Switzerland

\section{Dipl.Ing. Didier Chauvin on behalf of the W7-X Team.}

Abstract - Wendelstein 7-X (W7-X) is a fully optimized low-shear stellarator and shall demonstrate the reactor potential of this fusion plant. It is presently under construction at the Greifswald Branch Institute of IPP. The superconducting magnet system will allow continuous operation, limited only by the plasma exhaust system whose capacity is designed for 30 minutes full power operation. The Wendelstein 7-X (W7-X) coils and structures are part of the largest superconducting fusion device being constructed at present. They represent a technical challenge at industrial level and the need for proven techniques and manufacturing processes in accordance to the highest quality standards. The production of these components requires a management of monitoring for quality and tests. The coil system consists of 20 planar and 50 nonplanar coils. They are supported by a pentagonal $10 \mathrm{~m}$ diameter, $2.5 \mathrm{~m}$ high coil support structure (CSS). The CSS is divided into five modules. Each module consists of two equal half modules. The manufacturing status of the CSS and the main project management and technical challenges will be presented. The lessons learned in the large scale production of this difficult kind of support structure will be presented as relevant experience for the realization of similar systems for future fusion devices, such as ITER.

Key words: Manufacturing, coil support structure (CSS), half module (HM), flanges, design, process.

\section{Introduction}

The main supporting structure of the superconducting magnets of the Wendelstein $7-X$ Stellarator under construction in Greifswald consists of a stainless steel, AISI36 LN ring structure (11 $\mathrm{m}$ diameter, $2.5 \mathrm{~m}$ height, 72 tons), which is divided in five modules. Each module is subdivided in two half modules (HMs) for assembly reasons. The ring structure and its connections were designed before the start of the construction on the base of the results of a complex but partial structural analysis model, using anti-symmetric boundary conditions in order to limit the model size. Two robust vertical/radial flanges (sides of the module in Fig.1) bolted together with M45 studs will be used for the connection between modules.

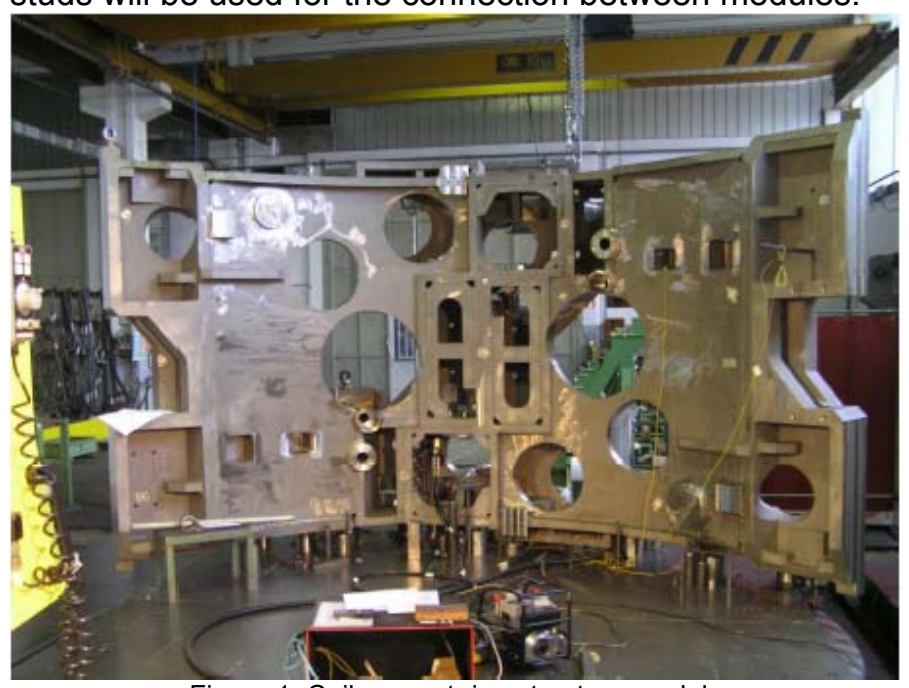

Figure 1. Coil support ring structure module.

The connection between the HMs, which has to resist higher loads, was designed using steel step flanges (left side of the $\mathrm{HM}$ in Fig. 2), mating with the corresponding step flange of the adjacent HM, and using M45 studs.

More accurate analyses using global models of the magnetic system found out (2004-2006) that the loads on the connections were much higher than previously computed. The load increase was mostly compensated by using high strength INCONEL 718 studs and tensioners.
However a component of the loads tends to warp the ring structure at the half module flange connection and for the most severe load conditions the flanges slightly slip and start to open.

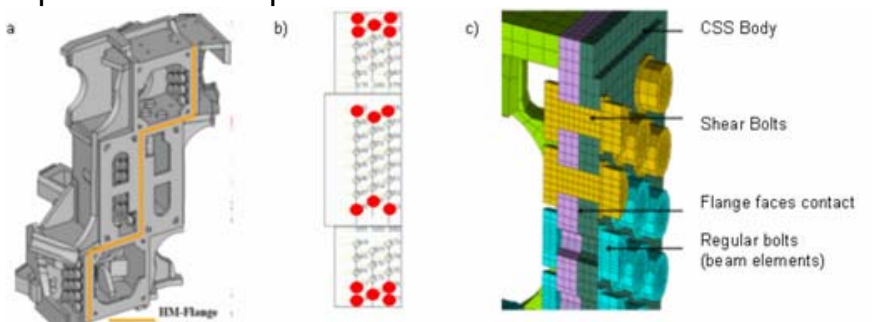

a) View of HM flange b) Shear bolt position sc) FE model section of flange at shearbolt location Figure 2. Shear bolt locations in CSS HM flange.

In order to solve the problem without radically changing the design of the support ring in advanced status of manufacturing, a solution has been proposed, which makes use of a selected number of studs as shear studs. In addition to the friction forces of the standard bolted connection, the shear studs resist the shear loads and limit the warping. The use of shear studs requires a precise mating with the flange holes. This is normally achieved by reaming together flange holes.

The development of the CSS has continued using full and reduced scale experiments and FE models. Design changes have already been implemented based on the outcome of the experiments and models

The CSS manufacturing has reached a phase where many support elements are being installed for the first time. This requires closure of several technical issues that the program faced in the recent years. The numerous design and test activities on the various support elements have provided a solid basis for ensuring that the CSS will perform reliably throughout the experimental life of the W7-X stellarator.

\section{Organisation of the manufacturing.}

The CSS's detail design has been subjected to considerable modifications over the years, since 1999. Thanks to a strong collaboration between IPP and several sub-contractors, the design of CSS has been 
continuously improving till it fulfils the requirement of dimensional accuracy, and also complies with the optimized capacity of production for casting, machining, welding and cleaning activities.

An industrial consortium by ENSA (Spain), ROVERA (Italy), ROMABAU-GERINOX (Switzerland), M\&G.srl (Italy), is participating in the manufacturing of the $10 \mathrm{HM}$ 's of CSS. Logistical tasks are one of the difficulties of the project, because during all manufacturing steps each $\mathrm{HM}$ has to cover almost $7000 \mathrm{~km}$ before being delivered at IPP Greifswald (see also in Fig.3).

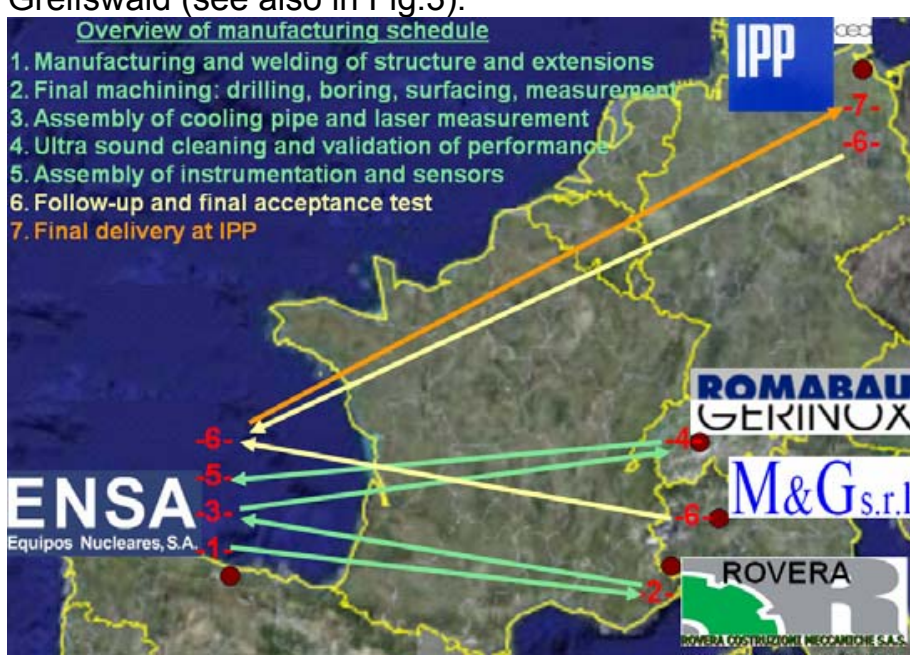

Figure 3. Overview of logistical tasks for CSS's manufacturing.

In order to compensate intrinsic difficulties due to the remoteness between different subcontractors, a clear documentation, the quality of drawings, work culture, a permanent communication and management are really fundamental to obtain a good quality of manufacturing which complies with delivery schedule. For improving the follow-up and advancement of manufacturing tasks, the CSS production is not only supervised by IPP but also supported by external inspectors. Indeed, the role of M\&G's inspectors is essential for following-up the contract in compliance with highest standards in quality assurance. The purpose of these inspections is also to validate respective rules, norms and regulations inherent in the step-by-step manufacturing of CSS. The experimental life of CSS manufacturing also concerns also the cleaning. In June 2008, the company Romabau-Gerinox was also involved to enhance the cleanliness of each HM by means of ultrasounds cleaning. This additional tasks has modified the organisation and of course the logistic of the project. This new organisation is described in Figure 4.

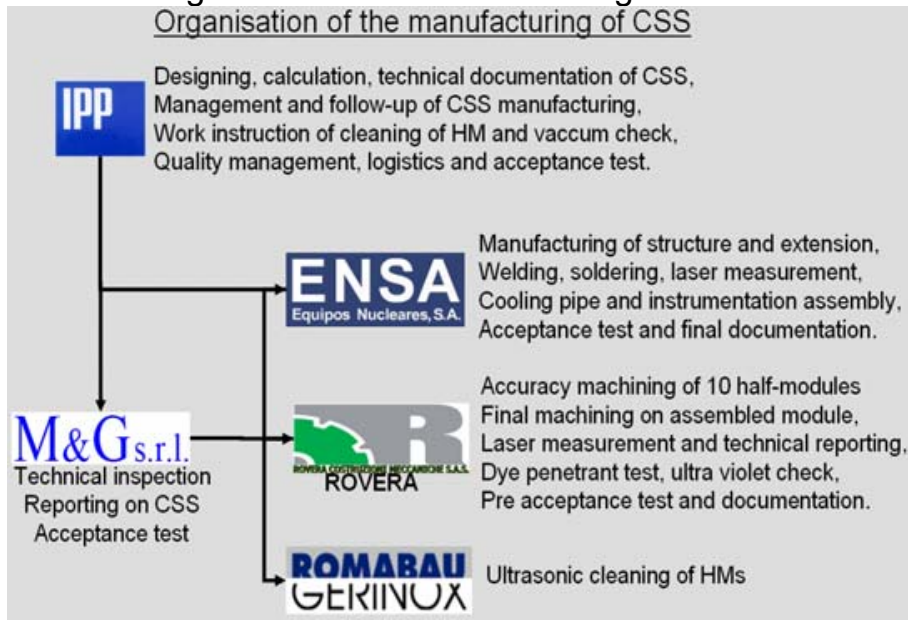

Figure 4. Organisation of the manufacturing of CSS.

\section{Calculation, design and manufacturing.}

The objective of the CSS calculation project was to create a finite element model of one half module and analyse its mechanical behaviour under loads resulting from three plasma scenarios with preliminary bolt tightening and cooling down from $295 \mathrm{~K}$ to $4 \mathrm{~K}$.

Finite element computations were carried out in order to obtain the complex stress distributions inside the CSS. The maximum stress and strain values can be kept within technical limits by the current design of CSS (see also in Fig.5 and 6) and the 3D design of HMs.

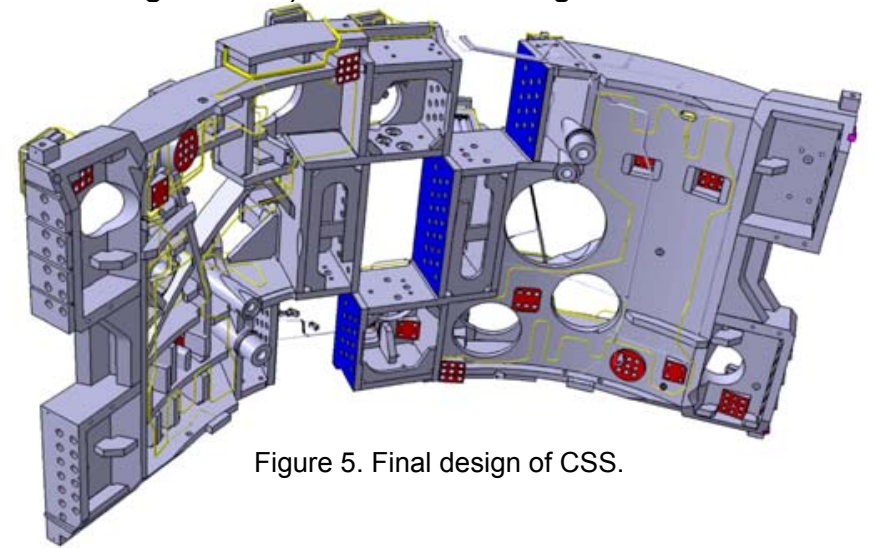

Finally, CSS has been developed to some extent but further optimization works were necessary in order to minimize the amount of structural material and to homogenize the mechanical behaviour of the CSS. Analysis of the CSS model showed sliding of around 0.5 $\mathrm{mm}$ on the top and bottom of the module flanges (flat flange) (see also in Fig.6).

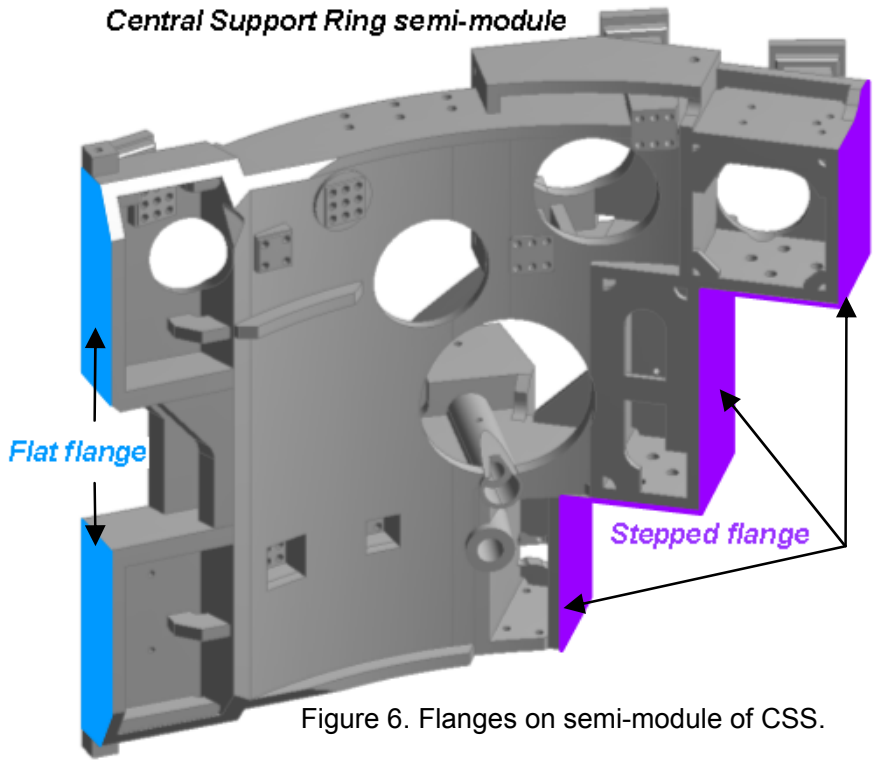

Over $1 \mathrm{~mm}$ was observed on the stepped flanges. The basic design of the flange connections was based on pure friction to take any shear loads exerted onto both the flat and stepped flanges. It was decided to add mechanical aid in the form of a radial locking key for the flat flange and a total of 16 shear bolts for the stepped flange. By means of analysis this has been performed assuming full relative fixation of the top and bottom ends of the flanges. This reduced sliding from 0.5 down to $0.29 \mathrm{~mm}$ in the region of highest sliding activity at the flat flange as described in Fig. 7. In addition, the CSR weld assessment showed high stresses in several regions of CSS. 


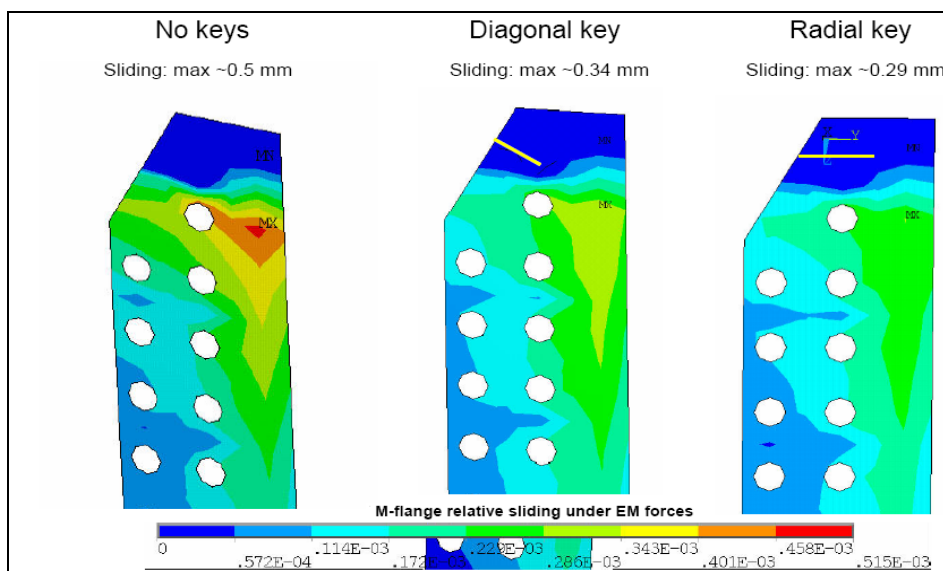

Figure 7. flat flange relative sliding under EM forces.

Reinforcement of the CSR has been assessed keeping in mind the restriction with respect to manufacturing and assembly. The main goal of the reinforcement was to provide a higher bending stiffness around the vertical axis for the module flange which was seen to be the weak point of the CSR. An evolution of the reinforcement configurations is shown in the Fig. 8
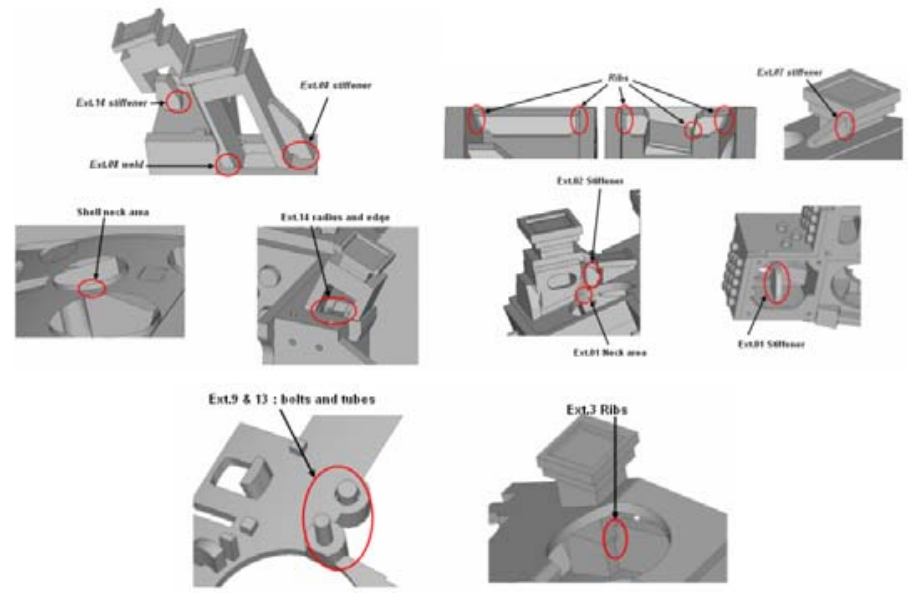

Figure 8. bolted regions for both flat and stepped flanges.

The analysis revealed several critical regions as shown upon. These regions where subjected to large stresses. Of special interest is the neck region near ext1 and ext2 as this "bottleneck" is subjected to large stresses and yielding could be a problem is this area. This result also started the requirement to assess possible reinforcement in this region.

The extensions of CSS provide the main support for all coils. All the central support elements are bolted connections where Superbolts $₫$ are screwed into the threaded holes on the DIN1.3960 CSE blocks on the coils, an example is shown in Fig 9

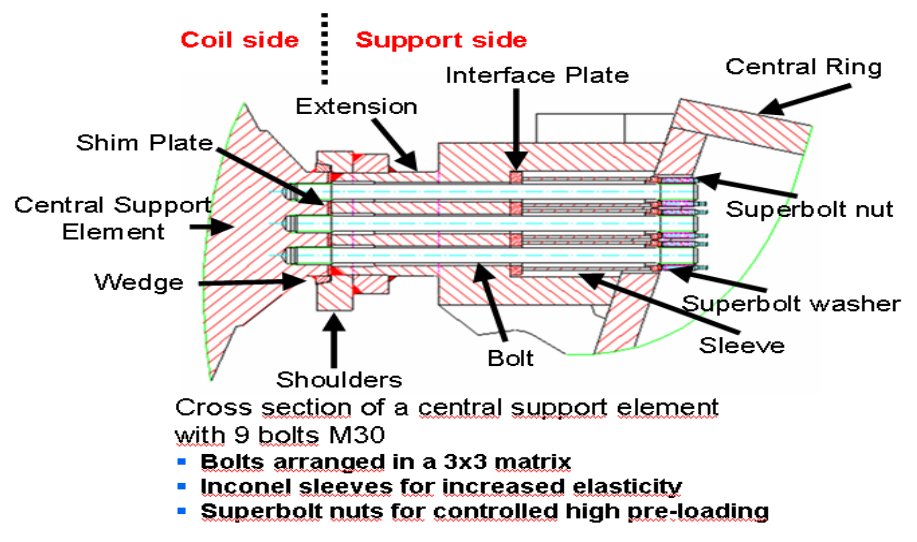

Figure 9. Connection of coil on "Fork" extension of CSS.
Tightening the bolts presses the flange faces from the CSS and coils together to form a frictional joint. Shoulders and wedges around the flange faces provide additional support against large shear and bending loads coming from the coils.

The development of the CSS has continued using full and reduced scale experiments and FE models. Design changes have been continuously implemented during manufacturing and are based on the outcome of the experiments and models.

\section{Technical specifications during manufacturing of CSS}

\subsection{Standards in quality assurance:}

IPP technical specifications inherent in the step-by-step manufacturing of CSS should comply with rules, norms and regulations. For instance, general tolerances are defined for linear and angular dimensions on norm DIN ISO 2768-m (see also picture in Fig. 10).

\begin{tabular}{|c|c|c|c|c|c|c|c|c|c|}
\hline \multicolumn{2}{|c|}{ Tolerance class } & \multicolumn{8}{|c|}{ Permissible deviations for basic size range } \\
\hline Designation & Description & $\begin{array}{l}\text { from } \\
0,5^{\circ} \\
\text { up to } \\
3 \\
\end{array}$ & $\begin{array}{l}\text { over } \\
3 \\
\text { up to } \\
6\end{array}$ & \begin{tabular}{|l} 
over \\
6 \\
up to \\
30
\end{tabular} & \begin{tabular}{|l} 
over \\
30 \\
up to \\
120
\end{tabular} & \begin{tabular}{|l} 
over \\
120 \\
up to \\
400
\end{tabular} & \begin{tabular}{|l} 
over \\
400 \\
up to \\
1000
\end{tabular} & \begin{tabular}{|l} 
over \\
1000 \\
up to \\
2000
\end{tabular} & $\begin{array}{l}\text { over } \\
2000 \\
\text { up to } \\
4000\end{array}$ \\
\hline$f$ & fine & $\pm 0,05$ & $\pm 0,05$ & $\pm 0,1$ & $\pm 0,15$ & $\pm 0,2$ & $\pm 0,3$ & $\pm 0,5$ & .. \\
\hline m & medium & $\pm 0,1$ & $\pm 0,1$ & $\pm 0,2$ & $\pm 0,3$ & $\pm 0,5$ & $\pm 0,8$ & $\pm 1,2$ & \pm 2 \\
\hline c & coarse & $\pm 0,2$ & $\pm 0,3$ & $\pm 0,5$ & $\pm 0,8$ & $\pm 1,2$ & \pm 2 & \pm 3 & \pm 4 \\
\hline v & very coarse & .. & $\pm 0,5$ & \pm 1 & $\pm 1,5$ & $\pm 2,5$ & \pm 4 & \pm 6 & \pm 8 \\
\hline
\end{tabular}

Figure 10. Permissible deviations for basic size range on CSS.

According to DIN ISO 2768-m general tolerances for linear measures and level squares with four tolerance classes are useful for simplifying drawings. By choosing the tolerance class precision levels common in workshops should be taken into account. If smaller tolerances are needed or bigger ones are more economical, then these tolerances are indicated next to the nominal size.

\subsection{Materials of CSS:}

Properties and stress/strain curves for each material are listed in the following sections. The material properties are defined at 295, 77 and $4 \mathrm{~K}$, and are linearly interpolated in between and the part material attribution is presented in Fig.11 and 12. The plates of the ring including reinforcement plates, the extensions and the shims are made of 1.4429 stainless steels (see also in Fig. 11).

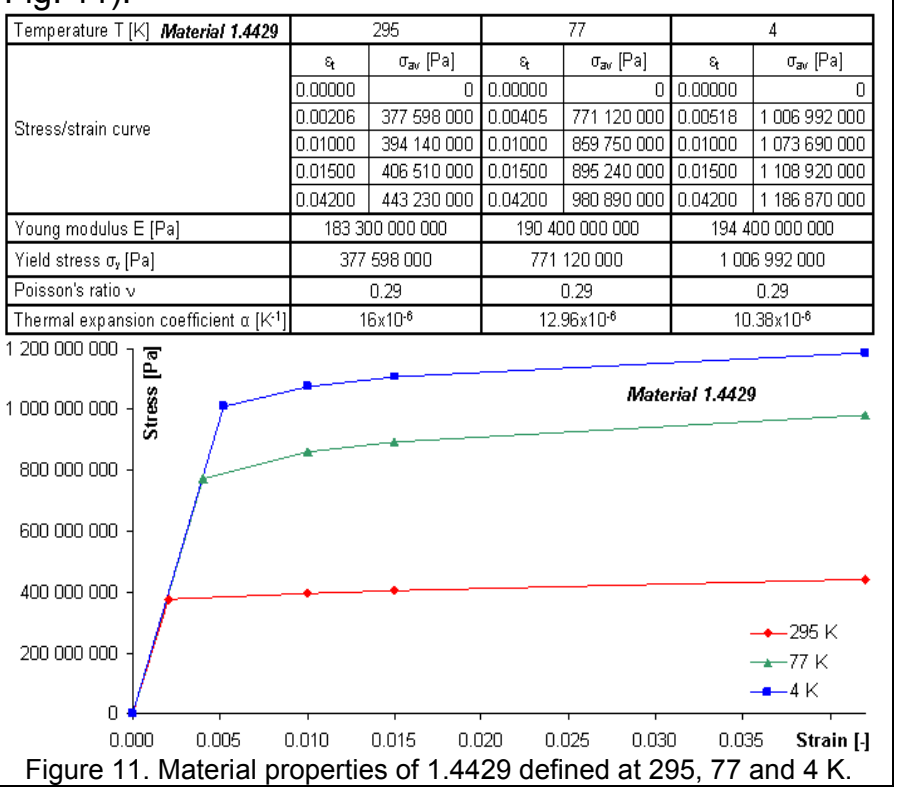


The casting of the ring is made of 1.3960 stainless steels (see also Fig.12).

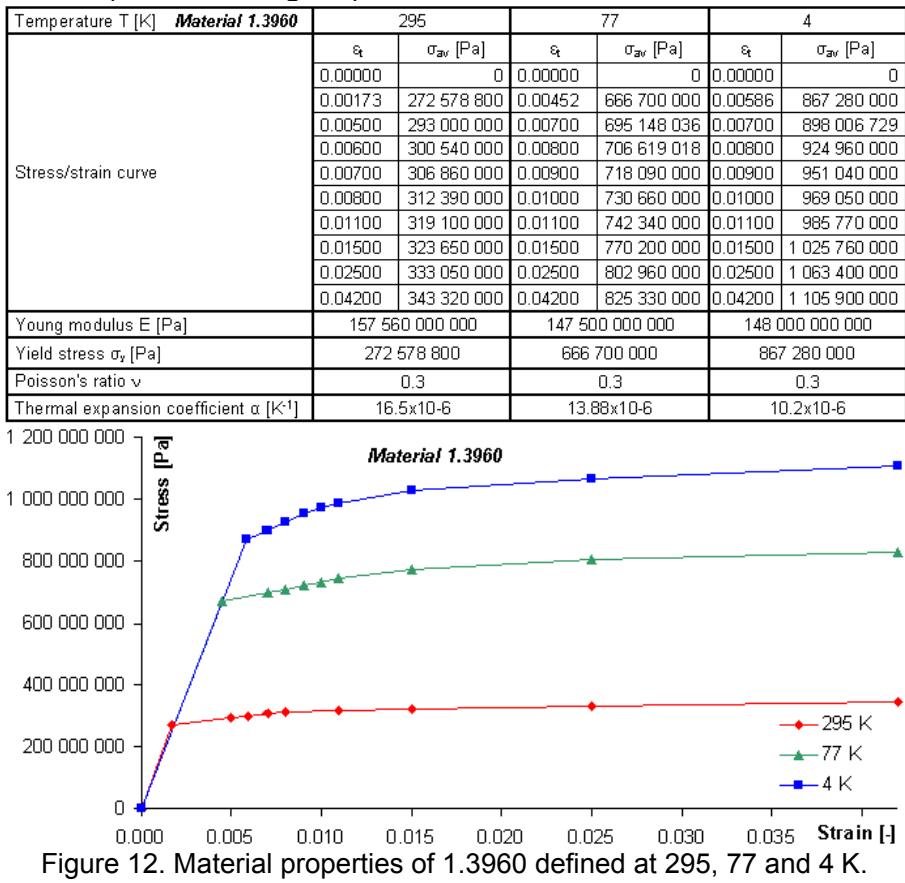

The part material attribution is defined in Fig. 13 The bolts, including nuts, studs and washers connected on CSS, are made of 2.4668 INCONEL 718 .

Material repartition

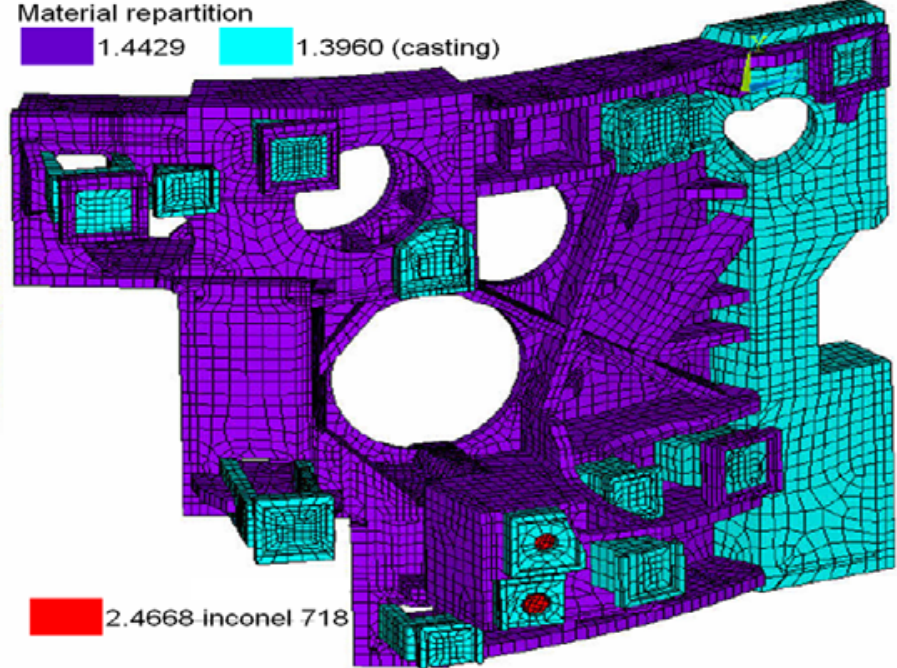

Figure.13. Part material attribution on half module.

On receiving all materials, semi finished products and prefabricated parts, the company ENSA and his subcontractors ROVERA had to issue certificates. All material and batch numbers and all needed properties must be contained in these certificates. In addition, IPP is performing an independent analysis concerning stainless steel's amount of 500 ppm of cobalt is to be carried out.

4.3. Welding works of HM's frame and extensions:

DIN EN 288 specifies how a welding procedure specification is qualified by welding procedure tests, ranges of qualification and acceptance levels. In that way, IPP and his subcontractors have developed all necessary welding qualified procedures according to requirements based on the related DIN/EN rules. These DIN rules describe the good practice work which has been performed by the companies. In IPP's supply contract it is requested to ENSA to work according to the scientific and technologic state of art. All along the manufacturing of each HM of CSS, several tests are performed : visual check, test of welding seems, measurement after heat treatment, permeability, US controls, dye penetrant test, $\mathrm{X}$-rays, etc. The purpose of these tests are necessary for detection of crack, surface pore, lack of fusion, incomplete root penetration, undercut (continuous and intermittent), shrinkage groove, excess weld material, excessive convexity, excess penetration, incorrect weld toe, overlap, burn through, asymmetry of fillet weld, root concavity or porosity, poor restart, insufficient throat, which are among the possible non-conformances detected during the test.

4.4. Extensions, ports and assembled accurate machining:

Accurate machining is performed by ROVERA. Since 2005, an important historical background was developed in order to improve the final machining of CSS. For technical and financial reasons of production a strong and permanent technical collaboration between IPP, ENSA and ROVERA is needed in order to:

- Propose and argue changes of the HM design related to technical difficulties and extra cost processes,

- For each of the processes involved, propose a process of estimates and principles associated tooling,

- Validate the choice of couple material/machining process with regard to the geometry and specifications for producing each HM,

- Specify the means of production requirements: machine-tool (gantry machine at ROVERA) tool support,

- Establish documents, procedures for machining and quality control (QIPP documentation).

The numerous design and test activities have provided a solid basis for the feedback of big piece like the 10 half module composing the central support structure. All along these technical experiences, the machining process had been then developed, manufactured and successfully tested. Accurate machining is now confirmed by measurement's report in sensitive area for holes of the three step flanges, extensions or module flanges as shown in Fig. 14.

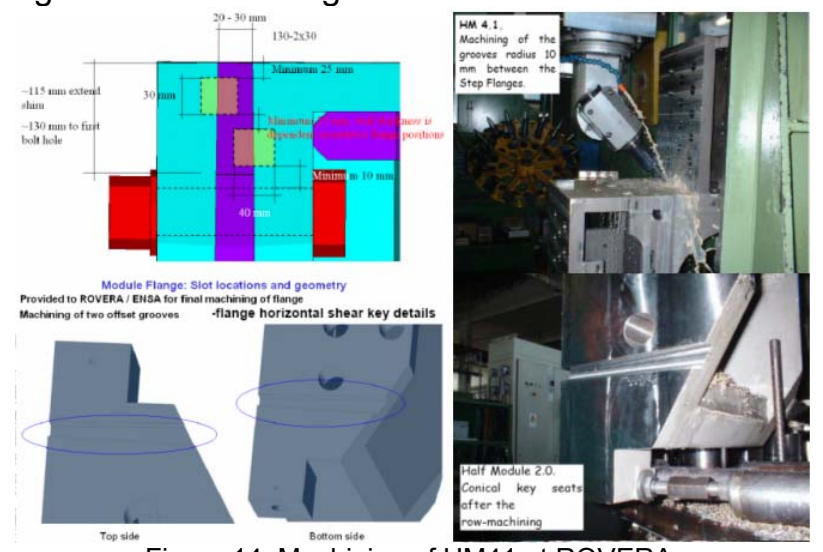

Figure 14. Machining of HM41 at ROVERA.

\subsection{Cooling pipe assembly:}

Bending, cutting, milling, welding, soldering and testing tasks are necessary for a complete cooling pipes assembly. Helium cooling pipes used during the manufacturing are seamless and from 1.4571 materials. For each half module, 45 meters of cupper strip (20x1) and 49 meters of 1.4571 pipes are needed. This task is performed by ENSA as demonstrated in Fig 15. The duration of cooling pipes activities is of 3 weeks, 
completed of helium leak detection. A rotative welding device is also used for connection of each pipe, but so far only visual check is done.

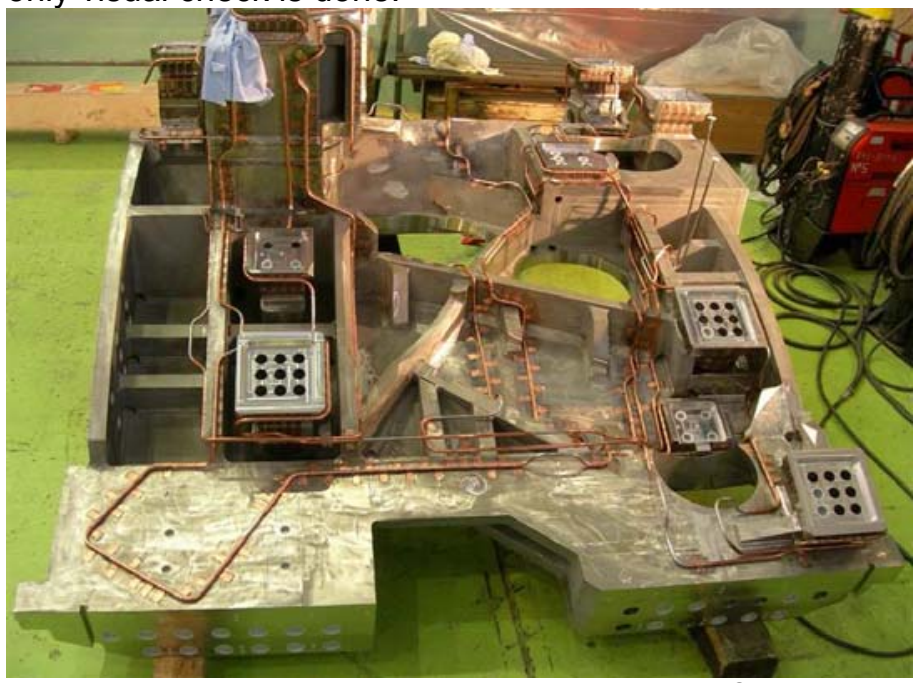

Figure 15. Helium cooling pipes assembly at ENSA.

\subsection{Measurement:}

The Laser-Tracker/Theodolite system is mainly used for adjustment and inspection of components composing each HM at ROVERA and ENSA. Therefore, well defined reference points, mostly fitted holes, and in minor cases punch cones, are used to determine the position or geometry of the CSS (see also in Fig.16).

measurement central ring by Rovera - critical areas

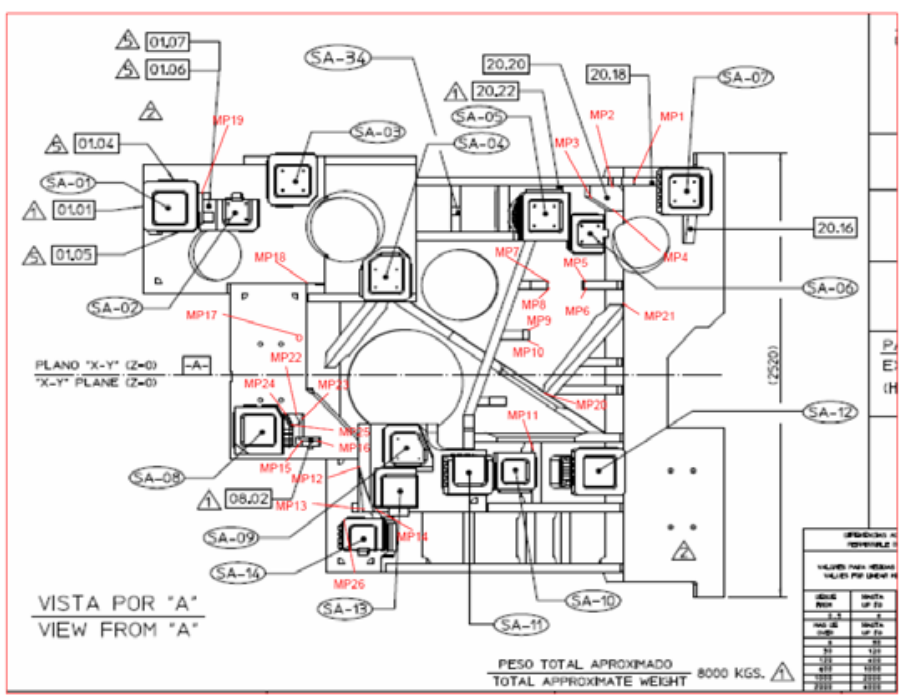

Figure 16. Measurement of HM-critical areas.

With a nominal measurement inaccuracy of $2 \sigma(95 \%)=$ $0.1 \mathrm{~mm} / \mathrm{m} \times L+0.05 \mathrm{~mm}(L=$ distance between measurement point and head of Laser-Tracker) the LaserTracker is an appropriate surveying tool to reach the above mentioned adjustment accuracies. Devices can be placed on the machine centre line for best line of sight allowing for the machine to be measured in the machine global co-ordinate system. Experience has shown that HM can be positioned within less than $1 \mathrm{~mm}$ of their theoretical position and re-positioned back to that position to within $0.4 \mathrm{~mm}$. These extremely stringent requirements mean that several metrology teams from ENSA and ROVERA will be required to operate simultaneously in order not to delay the progress of machining. Two laser measurements are done during the manufacturing of $\mathrm{HM}$, first before the final machining, the second for the acceptance test reporting. including also helium pressure test (30bars) and All the laser measurement points are presented on the Fig. 17.

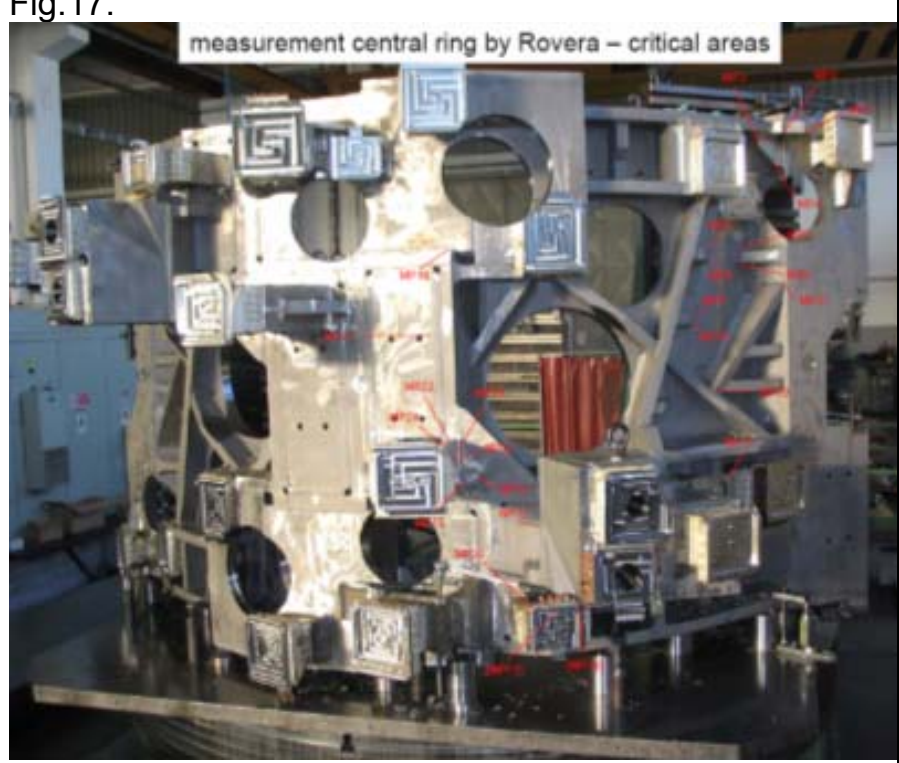

Figure 17. Status of measurement's points after machining.

\subsection{Ultrasounds cleaning:}

In contrast to all other large components of the W7X, the central support ring has no consistent surfaces but many faces that stand angular to each other. Some faces are hidden, a part of the surfaces is not machined (rough) and many surfaces are not accessible at all. Therefore, the cleaning media do not reach all surfaces during cleaning or run off badly. A first cleaning is performed at ROVERA after the final machining by the German company DERUSTIT. The purpose of this first middle level cleaning is to clean the surfaces verifiable from the media for removing most of oil or dust after final machining. After the drying process the surfaces are to be checked with an ultraviolet lamp visually for impurities possibly left behind and still visible. In addition, a smear test has to be carried out, particularly with regard to the holes and drillings and hardly accessible areas. Objective of spray cleaning is to reach surface conditions compatible with demands for next operation of soldering and cooling pipe assembly at ENSA. Nevertheless, this first cleaning does not allow technical requirement fixed by the vacuum usage with a pressure <1.10-6 mbar. To reach the required value it was decided in June 2008 to perform an additional cleaning after the last machining operation at ROVERA. The company ROMABAU-GERINOX is in charge of performing an ultrasounds cleaning in order to reach surface of difficult access. Difficulties are the following:

- Complex surfaces and closed volumes of HM.

- A large part of the surfaces is not machined (surface roughness $>6.3$ (sponge effect).

- $\quad$ There are copper-steel-combinations $\rightarrow$ oil's trap.

- During first cleaning the numerous holes and drillings have to be cleaned by immersion/dilution.

- HM's surfaces are contaminated with different media: Dye residue from the weld inspection, residual oil, sludge and grease from the machining at ROVERA.

- Important flux residue from the soldering at ENSA.

US cleaning was chosen for several reasons. ROMABAU- GERINOX uses generators, which produce high frequency electricity, create ultrasound. This high 
frequency electricity is then converted to mechanical energy or sound waves through a transducer, which literally makes these waves, vibrate. As these vibrating sound waves travel through water, microscopic bubbles form and repeatedly implode upon all given surfaces of CSS. This powerful action removes visible and even microscopic dirt particles making a dirty mini-blind or any other object cleaner than alternative methods. Not only is this ultrasonic cleaning method extremely effective, but also it is fast, safe, and gentle. The checking is done at IPP Greifswald by vacuum group (see also Fig.18).

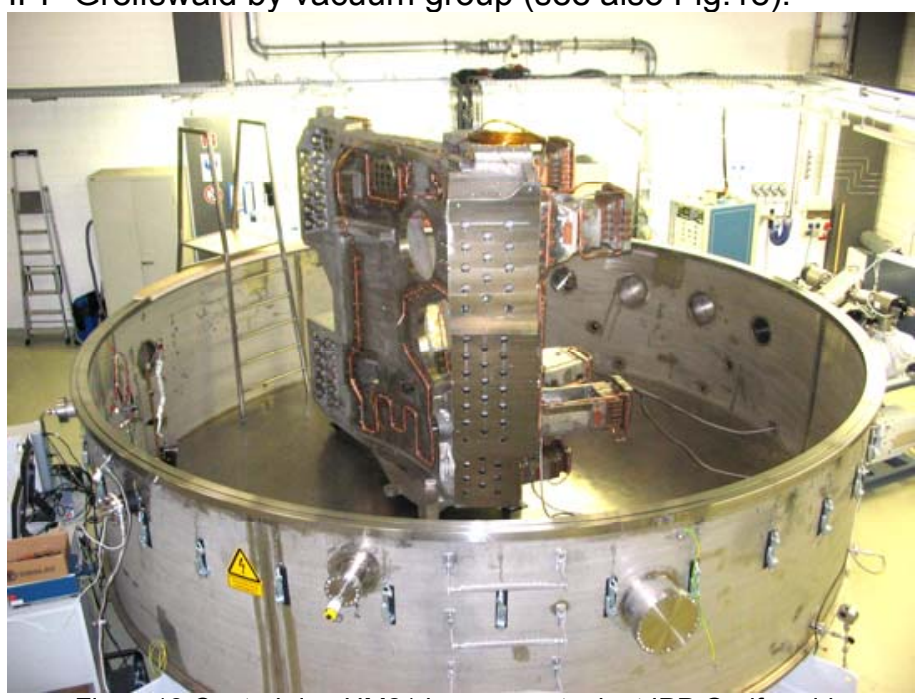

Figure18 Central ring HM21 in vacuum tank at IPP Greifswald.

For that purpose the rings will be installed in a vacuum chamber and a residual gas analysis will be carried out. The spectrum of the empty chamber is the reference. The spectrum of the ring should arise a collection of similar mass peaks with comparable intensity. If this spectrum does not show additional main peaks compared with the reference spectrum, the cleaning can be accepted.

\section{Results and conclusion.}

Because of a strong collaboration between IPP and several sub-contractors, the design of CSS has been continuously improved till it fulfils the requirement of dimensional accuracy, and also complies with the optimized capacity of production, machining, assembling and cleaning activities.

In order to compensate intrinsic difficulties due to the remoteness between different subcontractors, a clear documentation, the quality of drawings, the work culture, a permanent communication and management are really fundamental to obtain not only a good quality of manufacturing but a well manufacturing process. For improving the follow-up and advancement of manufacturing tasks, the CSS production is not only supervised by IPP but also supported by external inspectors.

The 1st and 2nd half modules had been completed and delivered to IPP at the beginning of 2007. Both were successfully assembled with the coils meeting the tight manufacturing tolerances.

Also the 3rd and 4th half modules were delivered to IPP in February 2008. The final machining of the 5th and 6th half modules has been successfully completed; both will be delivered in September 2008.
Therefore, the current feedback in the framework of international project management for designing and manufacturing of big component requires a large scale of general knowledge in the field of international action. Whether as the management of technical problems are arising from cultural differences, or on work culture on the daily operations of companies internationalized, the ability to communication and interpersonal skills are essentials. The feedback in designing and manufacturing of central support ring is a typical example of difficulties expected on ITER. The trade of responsible officer, in particularly in the field of big components manufacturing such as W7-X coil support structure involves not only, of course, technical and engineering skills but also versatility, good analytical capabilities, communication skills, organisational skills and ability to work under pressure in a multicultural international setting are prevailing.

\section{Didier Chauvin.}

Responsible officer for designing and manufacturing of the coil support structure of W7-X project at IPP Greifswald.

Service W7-X Magnete und Kryostat

Max-Planck-Institut für Plasmaphysik

Teilinstitut Greifswald

Unternehmung Wendelstein 7-X

Wendelsteinstraße. 1

D - 17491 Greifswald

Tel:+49 (0) 3834 / 882758

Fax:+49 (0) 3834 / 882709

e-mail: didier.chauvin@ipp.mpg.de 


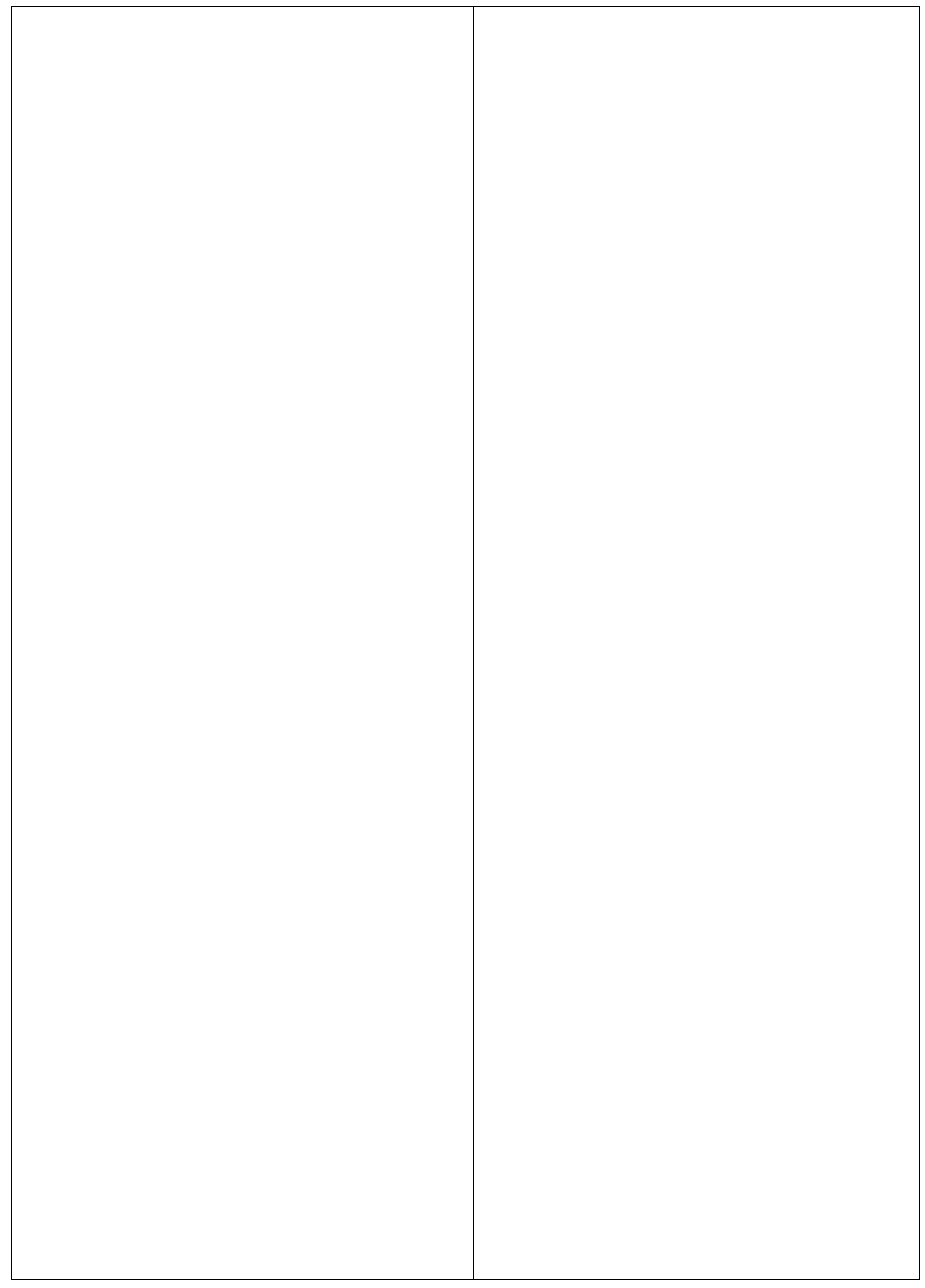


